\title{
El Realismo Crítico y su modelo transformacional de la actividad social
}

\author{
The critique realism and his transformational \\ model of the social activity
}

Guillermo Alfonso Guerra Madrigal Universidad Autónoma de Aguascalientes alfonsoguerra.m@gmail.com

\begin{abstract}
Resumen
En el presente artículo se pretende defender la ontología propuesta en el Realismo Crítico de Bhaskar, como base epistémica para el estudio del objeto en las ciencias sociales, dentro de la discusión metodológica entre Individualismo y Holismo, para lo cual, se defiende el modelo transformacional de la actividad social (T.M.S.A.), como un modelo que trata de explicar los procesos de socialización, reproducción y transformación de la sociedad.

Palabras Clave: Realismo Crítico; Individualismo; Holismo.
\end{abstract}




\begin{abstract}
In this article I try to defend the proposed ontology in Bhaskar's Critical Realism, as an epistemic basis for the study of the object in the social sciences, within the methodological discussion between Individualism and Holism, for which, the transformational model of social activity (TMSA), is defended as a model that tries to explain the processes of socialization, reproduction and transformation of society.
\end{abstract}

Key Words: Critical Realism; Individualism; Holism.

\title{
Introducción
}

El presente trabajo parte de una investigación sobre cuestiones epistémicas de las ciencias sociales, dentro del debate metodológico entre el individualismo y holismo. Para esto se analiza el papel que desarrollan las conceptualizaciones de socialización, reproducción y transformación de los fenómenos individuales, sociales e históricos. Se propone el modelo transformacional de la actividad social desarrollado por Bhaskar, que parte de su Realismo Crítico, anteponiendo la ontología a la epistemología, es decir la preeminencia del ser del objeto de estudio, frente a los modelos de dicho objeto de estudio.

La relación entre el individuo y la sociedad es parte fundamental de la teoría social, esta implica posiciones ontológicas y epistémicas, en la sociología las tradiciones metodológicas con enfoque holista o individualista siguen vigentes y nos remontan a sus 
principales fundadores siendo el caso del Holismo con E. Durkheim y el individualismo con la sociología comprensiva de M. Weber. Se analizan las implicaciones de los enfoques metodológicos del holismo y el individualismo, para ello se expone el realismo crítico, para la defensa de un modelo con base ontológica sobre el objeto de estudio con el fin de que sirva de explicación a la reproducción o transformación de los fenómenos sociales.

\section{¿Qué es el Realismo Crítico?}

El Realismo Crítico tiene por antecedentes trabajos de filosofía de la ciencia de filósofos ingleses, a partir de los trabajos de Rom Harré y Mary Hesse y otros realistas, continuados por Roy Bhaskar. Así, Bhaskar desarrolló el Realismo Trascendental (1975), una crítica a la tradición empirista del positivismo e idealismo trascendental; posteriormente, Bhaskar lo aplicó a la filosofía de las ciencias sociales (Naturalismo Crítico), en su obra, The Possibility of Naturalism: A Philosophical Critique of the Contemporary Human Sciences (1979); y por último desarrolló una epistemología política llamada Realismo Crítico Dialéctico (1993), en donde parte de la filosofía de la ciencia y se extiende a la filosofía política, con el fin ulterior de lograr una emancipación humana.

La filósofa Adriana Murguía señala que «los intereses a lo largo de la obra de Bhaskar son tres: el primero es restablecer una ontología realista, a través de argumentos trascendentales; el segundo, es realizar 
un análisis correcto de las ciencias naturales y sociales; y tercero, la posibilidad de una práctica científica y filosófica dirigida a la emancipación humana» (Murguía, 2006; 70).

El R.C. de Bhaskar desarrolla una crítica tanto al realismo empírico (positivismo), como al idealismo trascendental (constructivismo); en el cual hace un llamado a advertir la relevancia y necesidad de una ontología renovada, puesto que afirma que las cosas del mundo existen de manera independiente de nuestra experiencia y de nuestro conocimiento de estas mismas cosas, por el contrario la confusión sobre esta afirmación, constituye lo que él llama falacia epistémica, la cual es compartida por las posturas mencionadas, es decir, la tesis de que el ser puede reducirse a la percepción y lenguaje humanos, según Bhaskar esto es a lo que ha conducido a la disolución de la ontología realista originando un análisis equivocado de la praxis científica (Bhaskar, 2008; 28 - 35).

De esta manera la óptica del realismo crítico, consta de dos dimensiones del conocimiento en: 1) Transitiva: Los hombres en su actividad social producen conocimiento que es un producto social muy similar a cualquier otro, que no es más independiente de su producción y de los hombres que lo producen 2) Intransitiva: Es que el conocimiento es de cosas que no son producidas por hombres en absoluto: la gravedad específica del mercurio, el proceso de electrólisis, el mecanismo de propagación de la luz. Ninguno de estos «objetos de conocimiento» 
depende de la actividad humana. Si los hombres dejaran de existir, las estructuras causales seguirían existiendo; ya que, en su mayor parte son bastante independientes de nosotros (Bhaskar, 2008; 14).

Las objeciones de Bhaskar al realismo empírico y al idealismo trascendental es su ontología compartida, pues argumenta en contra del empirismo que los objetos de conocimiento son estructuras, no son sucesos de fenómenos, en contra del idealismo, tampoco son constructos humanos impuestos a dichos fenómenos. Según el realismo trascendental son estructuras reales y operan independientemente de nuestro conocimiento, nuestra experiencia y las condiciones que nos permiten acceder a ellos. En resumen, contra el empirismo, para los realistas trascendentales los objetos del conocimiento son estructuras, no sucesiones de eventos; contra el idealismo, son intransitivos, en el sentido antes definido.

La propuesta de Bhaskar es la reivindicación de la ontología, centrada en el objeto de estudio y no en el conocimiento sobre el objeto de estudio. Propone una ontología que favorezca al conocimiento, comprendido en la distinción de las dos dimensiones, transitiva e intransitiva, no reduce la ley causal a conjunciones constantes, como lo hace el empirismo positivista, asimismo le da importancia a la agencia humana para producir patrones de eventos para así, poder identificar los mecanismos que están presentes 
en la realidad, en la dimensión intransitiva. El mundo es ontológicamente estratificado por tres dominios: de lo a) real, b) actual y c) empírico:

a) El dominio de lo real consiste de estructuras y mecanismos reales con poderes causales. El Realismo Crítico es una filosofía pluricausal, esto es, hay causas físicas, biológicas, psicológicas y sociales. La pluralidad causal se explica por el emergentismo, que sostiene la existencia de múltiples estratos de realidad, irreducibles entre sí y con poderes causales.

b) Lo actual consiste de conjunciones constantes o patrones de eventos. Las estructuras existen independientemente de (y fuera de fase con) los patrones de eventos. Los patrones de eventos son generados por aquellas estructuras bajo ciertas condiciones.

c) Lo empírico se refiere a eventos percibidos por los seres humanos. (Afirma que solo pueden obtenerse patrones de eventos en sistemas cerrados, esto es, donde ciertos mecanismos causales han sido aislados de otros mecanismos que podrían inhibir su poder causal, tal como efectivamente sucede en los sistemas abiertos. El mundo real es un sistema abierto y la identificación de secuencias de eventos exige en general cerrar artificialmente el sistema, lo que es posible fundamentalmente por actividad experimental (Bhaskar, 2008; 47- 48).

Bhaskar menciona que el realismo empírico es la posición opuesta del realismo trascendental, ya que defiende una ontología basada en los límites de la experiencia humana y la tecnología; hace una 
reducción de lo real a lo actual y de lo actual a lo empírico. Por lo tanto, presupone un mundo cerrado y una ciencia completa de cómo es el mundo.

Empirical realism depends upon a reduction of the real to the actual and of the actual to the empirical. It thus presupposes the spontaneity of conjunctions and of facts. And in doing so presupposes a closed world and a completed science. (El realismo empírico depende de una reducción de lo real a lo actual y de lo actual a lo empírico. Por tanto, presupone la espontaneidad de las conjunciones y de los hechos. $\mathrm{Y}$ al hacerlo presupone un mundo cerrado y una ciencia completa) (Bhaskar, 2008; 49).

Los dominios de la realidad entendidos como real, actual y empírico conciben al mundo como un sistema abierto, donde los mecanismos y estructuras operan de manera transfactual, es decir, siempre están sucediendo sin considerar los resultados observables o no, aunque el mecanismo generativo, que tiene su base objetiva en la dimensión intransitiva, que constituye al mundo como sistema abierto, sólo ocurren actuando si las condiciones son adecuadas, ya que se encuentra en un sistema cerrado de laboratorio, para que puedan ser detectados los mecanismos generativos que actúan, sean o no observables.

La propuesta de Bhaskar para las ciencias sociales parte de sus fundamentos de su Realismo Trascendental, ya que pretende resolver una serie de dicotomías predominantes en las ciencias sociales, 
como es el híper naturalismo positivista y el antinaturalismo hermenéutico. El híper naturalismo positivista, se basa en la caracterización de Durkheim sobre los hechos sociales, estos con una realidad exterior a los individuos, por lo que se deben de tratar como cosas, es decir, objetos que tienen el mismo estatuto ontológico que los objetos del mundo natural, de esta manera son susceptibles de ser analizados por la ciencia.

La tradición naturalista en sociología presenta que los objetos de las ciencias naturales y sociales pueden metodológicamente ser considerados del mismo tipo. Por su parte, el anti-naturalismo hermenéutico caracterizado tradicionalmente por la sociología comprensiva de Weber, presenta una diferencia de objeto de las ciencias naturales y sociales que radica en que el objeto de las ciencias sociales es esencialmente de carácter significativo y preinterpretado, por lo que le corresponde un método interpretativo distinto al de las ciencias naturales. En cambio para el naturalismo crítico la realidad de los hechos sociales son diferentes, es decir, son emergentes de la acción individual, además sostiene la unidad de método cuyo objetivo es la explicación causal y descubrimiento de leyes (Murguía, 2006; 59).

Ahora bien, si tomamos la ontología de Bhaskar y las distinciones de los dos lados del conocimiento (objetos transitivos e intransitivos), encontramos una imposibilidad de dicho esquema para las ciencias sociales, por el objeto de estudio, pues es aquí lo social. Por lo tanto, si la intransitividad se refiere a una 
realidad independiente de la actividad humana, esto no se puede aplicar a los fenómenos sociales, ya que lo social es precisamente producto de la actividad social y parte del conocimiento humano, lo cual constituye principalmente la crítica al naturalismo (Murguía, 2006; 74).

Contra el positivismo, además de lo que ya se mencionó: en reducir lo ontológico a lo experimentable, Bhaskar no defiende la unidad de método con su tradicional posición positivista, en la que la unidad de método consiste de forma muy tosca a: «Observación + Correlación = Explicación + Predicción». Acher comenta que el realismo crítico no es del todo naturalista en la manera positivista.

Como Roy Bhaskar ha señalado a menudo, su libro también podría titularse La imposibilidad del naturalismo, ya que no defiende la unidad de método si esto se toma como sinónimo de «unidad metodológica», en la tradición positivista. Este último puede representarse crudamente como «Observación + Correlación = Explicación + Predicción» ${ }^{1}$.

${ }^{1}$ As Roy Bhaskar has often remarked, his book could just as well be entitled The Impossibility of Naturalism for it is not advocating the unity of method if this is taken to be synonymous with a «unity of methodology» in the positivist tradition. The latter can be crudely represented as "Observation + Correlation = Explanation + Prediction" (Bhaskar, Archer, Collier, Lawson, 2001; 190). 
En ciencias naturales es posible hacer la distinción de la dimensión intransitiva y la dimensión transitiva, pero en ciencias sociales la relación del sujeto que investiga es a la vez objeto de estudio, por lo que no es posible operar igual. Parada Corrales ilustra la distinción del empirismo y la relevancia de las estructuras y mecanismos en el realismo crítico:

Cuando un científico hace un experimento, está trabajando en un sistema cerrado, buscando un cierto mecanismo según el cual puede decir que A causa B, como en la causalidad de Hume. Pero en la realidad, no solamente en la sociedad, también en la naturaleza, hacemos frente a sistemas abiertos, cuando todos los factores están cambiando al mismo tiempo. Los experimentos son «artificiales» solamente en el sentido que los utilizamos para descubrir estos mecanismos en acción, para descubrir qué sucede en realidad allí afuera. En el realismo crítico, uno no permanece en el nivel de los hechos empíricos (lo que se observa), o en el nivel de acontecimientos o lo que sucede (eventos), uno se mueve en la dimensión de lo real, buscando las estructuras y los mecanismos causales ( Corrales, 2004; 415).

En resumen el Realismo Crítico, R.C. propone una ontología distinta a la del realismo empirista, sobre la que se basa el positivismo y el constructivismo, el mundo estratificado del R.C. pretende ser una ontología no antropocentrista, ya que diferencia a los objetos de estudio (intransitivos) de los objetos del 
conocimiento, que tratan sobre los objetos del mundo (transitivos) mostrando una posición más crítica ante las teorías y modelos en relación con la realidad del mundo.

Por esto mismo se muestra el mundo como un sistema abierto, las estructuras y mecanismos causales son reales y existen sin importar que las podamos percibir o no, la tarea del científico consiste en determinar dichas estructuras y mecanismos. Además en el realismo crítico los humanos son agentes causales, es decir las acciones de los agentes posibilitan acciones y actividades reproductoras o transformadoras.

\section{Entre el Individualismo y el Holismo}

En el siglo XIX ya estaba articulado el debate entre el individualismo y colectivismo. El individualismo es un enfoque metodológico que consiste en analizar a la sociedad desde la subjetividad, esto es, la parte psicológica donde se da relevancia al significado y sentido en el actuar de las personas, dando primacía al individuo respecto al colectivo. Se suele decir por tanto que es una metodología reduccionista. En Mill podemos observar una posición individualista, así la sociedad se puede reducir al individuo, por sus actos que corresponden a un cierto tipo de naturaleza humana; por lo que el individuo es el constituyente ontológico de la sociedad, mientras el colectivismo niega tal reducción como lo muestra Comte, la sociedad no es el conjunto de individuos. Es esto último lo que pretende 
demostrar el realismo crítico, de la relación entre individuos emerge la sociedad, como un ente distinto ontológicamente no reductible a sus partes.

Así para J.S. Mill, «Los hombres en un estado de sociedad siguen siendo hombres. Sus acciones y pasiones obedecen a las leyes de la naturaleza humana individual. Los hombres, cuando se juntan, no se convierten en otro tipo de sustancia con propiedades diferentes». En cambio, para Comte, «La sociedad no es más descomponible en individuos que una superficie geométrica en líneas, o una línea en puntos) $»^{2}$.

El individualismo metodológico plantea que todos los fenómenos sociales son reducibles a características de los individuos. Así pues, el individualismo se pude concebir como teoría explicativa, que además se articula con una tesis ontológica opuesta al holismo ontológico, por lo tanto, cuando se refiere a la forma en que se constituye la sociedad los todos sociales son considerados una falacia, no son otra cosa que construcciones, tal como fueron planteados por los

2 Thus to J.S. Mill, «Men in a state of society are still men. Their actions and their passions are obedient to the laws of individual human nature. Men are not, when brought together, converted into another kind of substance with different properties» (Mill, 1884; 573). Conversely, for Comte "Society is no more decomposable into individuals than a geometrical surface is into lines, or a line into points» (Comte, 1951; 181) (Bhaskar, Margaret Archer, Andrew Collier, Tony Lawson, 2001; 191). 
conceptualistas medievales en la discusión de los universales.

Para el enfoque individualista la perspectiva de las totalidades no existen en la realidad del mundo social, son modelos mentales del investigador. De tal forma, que toda institución o entidad social es configurado como un modelo ideal que sirve de explicación, es decir, es la forma de interpretar los productos de las relaciones abstractas de los individuos, los cuales son los constituyentes de la sociedad. Por esto mismo lo que se pretende estudiar son las interrelaciones entre los individuos con sus significados, creencias y disposiciones que dan sentido a su actuar. Las explicaciones macroscópicas de gran escala en la sociedad se pueden deducir a partir de los individuos, estos son los referentes de lo social.

Así pues el individualismo puede entender de forma epistémica a los fenómenos sociales como la explicación y comprensión del conjunto de acciones individuales, igualmente queda la ontología de las entidades sociales como la sumatoria o conjunto de las entidades individuales. Por lo que metodológicamente se suele inferir de las creencias empíricas más básicas de individuos concretos. Ejemplo de esto es la sociología compresiva de Weber:

Llamamos comunidad a una relación social cuando y en la medida en que la actitud en la acción social - en el caso particular, por término medio o en el tipo puro se inspira en el sentimiento subjetivo (afectivo o tradicional) de 
los partícipes de constituir un todo. Llamamos sociedad a una relación social cuando y en la medida en que la actitud en la acción social se inspira en una compensación de intereses por motivos racionales [...]. Entonces la acción, cuando es racional, está orientada a) racionalmente con arreglo a valores: en méritos de la creencia en la propia vinculación; b) racionalmente con arreglo a fines: por la expectativa de la lealtad de la otra parte (Weber, 1964; 33).

M. Weber sugiere el otorgarles nombres a esos fenómenos sociales para clasificarlos y poder estudiarlos, pero la existencia solo puede entenderlos como conjunto de individuos agrupados y clasificados, dependiendo de las interacciones y normas de las relaciones que hay entre ellos, así la conceptualización es no existente, solo existen los individuos en tanto agentes de la acción social. Weber, a diferencia de E. Durkheim y K. Marx asigna al estudio la importancia de la comprensión de del sentido subjetivo de la acción social.

Weber comenta en su teoría de las categorías sociológicas que algunos de los conceptos son construcciones, como conceptos-tipo, para poder entender la acción y en especial en el estudio de la historia, es por eso que estas abstracciones sean relativamente conceptos vacíos frente a la realidad concreta de lo histórico (Weber, 1964;16).

Por otra parte el holismo metodológico se contrapone al individualismo, plantea posiciones 
totalitarias o esencialistas, lo que se articula con una determinada ontología, por lo que según el holismo ontológico los fenómenos colectivos tienen características propias, autónomas y no son reducibles a sus componentes individuales, se sigue el principio de los sistemas complejos: el todo sería mayor o distinto a la suma de sus partes.

El holismo entiende que la conducta en gran escala está determinada por regularidades sociológicas, como estructuras o mecanismos. En el realismo crítico se habla de estructuras y mecanismos causales y se deja a un lado el término de leyes, pues ya que el mundo es un sistema abierto se prefiere a hablar de tendencias, por la característica de ser transfactual. Las tendencias de los fenómenos sociales en el holismo no pueden ser explicadas como meras tendencias que resultan de la conducta de individuos en interacción.

Para el holismo la conducta individual debe ser explicada, socialmente, en términos de macro regularidades, es decir, el todo no resulta de la sumatoria de las partes sino que es un producto diferente a las relaciones abstractas, como es el caso del emergentismo que defiende el realismo crítico así pues, las estructuras y mecanismos son considerados existentes en su propio nivel de estratificación ontológico. Las propiedades del todo son emergentes y no pueden reducirse a las propiedades de los elementos componentes. Como veremos más adelante en el modelo de Bhaskar, tiene bases marxistas, como es la tesis de K. Marx sobre Feuer Bach según la cual el individuo es en última instancia por el conjunto de sus 
relaciones sociales entendidas como relaciones socialmente construidas. Asimismo, se opone al reduccionismo individualista que estigmatiza a la totalidad social como una forma figurada de hablar y por tanto no como una entidad real.

El modelo de Durkheim es holista, siguiendo a Durkheim, el objeto de estudio de lo social son los hechos sociales, cuya ocurrencia y efectos no pueden ser reducidos a la simple suma de las acciones individuales. Durkheim al final de la primera parte: ¿Qué es un hecho social? de su obra: Las Reglas del Método Sociológico (1895), da una definición suficientemente abarcadora de las características que menciona en dicho apartado, de la siguiente manera:

Un hecho Social es toda manera de hacer, establecida o no, susceptible de ejercer sobre el individuo una coacción exterior; o también, el que es general en la extensión de una sociedad determinada teniendo al mismo tiempo una existencia propia, independiente de sus manifestaciones individuales (Durkheim, 2001; 51-52).

Desde una posición realista el sociólogo Emile Durkheim sostiene que es innegable el carácter real y colectivo de los hechos sociales, los cuales se basan en creencias y tendencias que se traducen en prácticas que se reflejan en el individuo. «De esta manera, individuos perfectamente inofensivos en su mayoría, pueden, reunidos en multitud, dejarse arrastrar a hacer cosas atroces» (Durkheim, 2001; 43). 
Durkheim entiende a la sociedad como un organismo, además de seguir al positivismo de Comte, también se vio fuertemente influenciado por el organicismo de Spencer, rechazando el psicologismo de Mill y con esto al individualismo. El organicismo es una aportación de la biología, así la sociedad es una entidad en sí misma y no un conjunto de personas individuales, pues los individuos son órganos que efectúan una función en la sociedad. Durkheim desarrollo una analogía con el mecanicismo del cuerpo señalando lo que podría ser perjudicial para una sociedad como patológica y el correcto funcionamiento como algo normal para una sociedad en específico.

En el enfoque del estructuralismo hay una mecanización que corresponde a las estructuras sociales, esto es el desempeño de una función por parte de los individuos, que fungen como órganos para la cohesión y permanencia de la entidad social, reconociendo un tipo de teleología en la sociedad que explica las funciones de los hechos sociales, los cuales son externos al individuo, son anteriores al individuo y ajenos de su consciencia, por lo que se dan de manera generalizada, independientemente de las manifestaciones individuales, pues se imponen de manera coercitiva en la sociedad.

Las metodologías con bases históricas e interpretativas éstas dan un mayor peso a la subjetividad y fuerza de acción del individuo, como es el caso de la sociología comprensiva de Weber, aunque él no desarrollo una metodología sistemática como Durkheim en Las reglas del método sociológico (1895), 
Weber a lo largo de sus investigaciones fue desarrollando una metodología que podríamos clasificar en el perímetro del instrumentalismo teórico por algunas de sus propuestas metodológicas.

La perspectiva de Weber se origina en una especie de síntesis de dos corrientes contrarias al estructuralismo de Durkheim, se trata de la discusión metodológica centrada en el objeto de estudio entre el historicismo y el neokantismo, lo cual ha demarcado el debate entre explicación (Erklären) y comprensión (Verstehen). El historicismo supone una distinción con las tesis cientificistas del positivismo para el conocimiento de fenómenos históricos y sociales, los representantes más destacados fueron Wilhelm Dilthey y Johann Gustav Droysen, así como los neokantianos de Baden: Wilhelm Windelband y Heinrich Rickert.

Las discusiones que se originaron el siglo XIX han tenido repercusiones en el siglo $\mathrm{XX}$ sobre el método para las ciencias sociales, así como los valores epistémicos de dichas ciencias. El historicismo abarca las primeras formulaciones sobre el Verstehen que fundamentalmente rechaza el monismo metodológico, es decir, la creencia de que las ciencias de la naturaleza que tienen por modelo las físico-matemáticas, según estas son útiles para todo tipo de conocimiento de la realidad, dicha tesis es compartida por el neokantismo; en segundo lugar la distinción consiste en que las ciencias de la comprensión (culturales) no pretenden establecer generalizaciones explicativas, sino la comprensión de los aspectos individuales, puesto que el ser humano es el objeto de estudio y al mismo tiempo 
el sujeto cognoscente, puesto que ambos se encuentra en el mismo tejido histórico y cultural en distinción clara en con el objeto de conocimiento de las ciencias naturales.

El Verstehen comprende al ser humano como una trama de relaciones significativas con su medio, el cual está compuesto de forma histórica y social, esto es construido de manera conceptual, por lo que las manifestaciones de los fenómenos sociales son resultado de la libertad y voluntad humana, en distinción del Erklären que pretende dar explicaciones en base a la necesidad natural, en esta diferencia radica la discusión metodológica entre la explicación y la comprensión que argumenta en contra o favor del monismo metodológico.

Para Weber la sociedad está compuesta fundamentalmente por los individuos, estos poseen intencionalidad y racionalidad y se relacionan entre sí, dando lugar a la acción, la mejor forma de analizar la acción es por la contracción de sus componentes y la base de esa acción es la acción individual, así la sociedad no es más que el conjunto de interacciones individuales en situaciones concretas. El investigador trata de comprender el significado de las acciones para dar una explicación causal a través de los objetivos y medios que el individuo dispone. Con esto realizó una dura crítica a las concepciones holistas que dan una existencia propia a la sociedad, como una cosificación del individuo como parte funcional de una entidad con conciencia propia, como es el caso del estructuralismo funcionalista de Durkheim. Weber apoya la idea de 
compresión de la sociedad a través de la probabilidad en distinción con Durkheim que debe ser estadística (Rodríguez, 2003; 47-48).

La metodología de Weber en relación con lo social entiende una distinción del ser en el objeto de estudio, es por lo que se pretende entender e interpretar la acción humana $y$ no según metodologías puramente naturalistas, esto en el sentido de estudiar a la sociedad y sus fenómenos con metodologías empleadas por las ciencias naturales y medios empíricos. Weber destaca la utilidad de los tipos ideales como instrumentos heurísticos.

Los tipos ideales constituyen una herramienta para el estudio de lo social, haciendo de los fenómenos interpretaciones a través de conceptos, como poder y burocracia; así los conceptos son creados por el científico social. Los tipos ideales no existen en la realidad concreta, son instrumentos conceptuales en base a los intereses explicativos y heurísticos. La finalidad del tipo ideal es ordenar la realidad para interpretar la realidad. Así mismo el investigador es demarcado por su contexto histórico, y psicológico de su espacio- tiempo (Gomez, 1986, pp. 282-286).

Para exponer las distintas metodologías entre el estructural funcionalismo que emplea Durkheim y los análisis de sentido que emplea Weber con su metodología de sociología compresiva, en relación con las diferencias entre católicos y protestantes, es destacable el estudio que hace Durkheim sobre el suicidio, ya que según las estadísticas recopiladas en dicho estudio hay una mayor cantidad de suicidios en 
protestantes que entre los católicos. Durkheim afirma que esto es porque las sociedades protestantes son de carácter mayormente individualista que las católicas, la razón que liga esta conclusión es la característica de libre examen en la confesión protestante, donde se genera una menor cohesión entre los creyentes, mientras los católicos al pertenecer a una iglesia más firme en su preceptos, hay una mayor cohesión entre los fieles.

Durkheim caracteriza dichos suicidios del tipo egoísta en las comunidades protestantes y muestra la siguiente estadística:

Promedio de suicidios por un millón de habitantes:

Estados Protestantes 190

Estados mixtos (protestantes y católicos) 96

Estados católicos 58

Estados católicos griegos

(Durkheim, 2014;pp. 139-147).

El punto central de la discusión es el objeto de estudio, nos centramos en la ontología de lo social, para así poder desarrollar una epistemología. Conforme entendamos el ser de los fenómenos sociales es cómo será la metodología de estudio. Así pues surgen preguntas que giran en torno a ¿Qué es la sociedad? y de aquí se desprenden otras, como: ¿Cuál es la metodología a emplear para dichos objetos?, ¿Existen esos objetos en la realidad del mundo o son construcciones conceptuales de nuestra experiencia? 
Para Weber la metodología de la sociología es a trasvés de la construcción de tipos ideales, los cuales son construcciones teóricas con el fin de hacer comparaciones y explicaciones, aunque se emplean registros estadísticos y pruebas de material empírico, no son tipos sociales empíricos, es decir, no existen en el mundo, solamente son una herramienta conceptual que sirve para hacer hipótesis contrastables con el mundo real de las acciones humanas. «Cuanto con más precisión y univocidad se construyan estos tipos ideales y sean más extraños en este sentido, al mundo, su utilidad será también mayor tanto terminológica, clasificatoria, como heurísticamente» (Weber, 1964; 17).

«Weber señalo que las distinciones entre objetos de estudio de las ciencias naturales y sociales no deben de hacer mermar la objetividad, así los tipos ideales se deben construir en base a juicios de hecho y no de valor» ( Rodríguez, 2003; 49).

La explicación de Durkheim es holista y así las acciones individuales son determinadas de cierta forma por el colectivo al que pertenecen, por lo que las acciones individuales como el suicidio tiene una relación directa con la integración de una sociedad. En cambio para Weber la acciones de los individuos se explican a través de los factores subjetivos que dan una explicación racional de los motivos y fines por los cuales actúa un individuo, por lo que el individuo no solo es un órgano que actúa conforme a una necesidad de cohesión social o a través de lo que le dicta la conciencia social, sino que es un ser dotado de libertad y voluntad, además el hecho de que sus acciones sean 
racionales las hace posibles de explicación y comprensión.

El enfoque de la sociología comprensiva busca entender los fenómenos sociales desde las posibilidades de las ciencias tanto naturales como sociales. La teoría de la comprensión de Weber pretende ser adecuada tanto causalmente como significativa, pues la causación y significación se relacionan, la comprensión causal se centra en el desarrollo histórico donde no se reduce a tratamiento estadístico, sino, que toma importancia los factores psicológicos como son los valores e ideas y creencias. Además la acción humana en este enfoque es entendida como un acto de sentido subjetivo, es aquí, la distinción entre sociología y psicología, sin embargo, para Weber «la acción social es una especie de conducta que implica un sentido para el actor mismo» ( Sandoval, 1986; 280).

Weber es claramente individualista en cuanto al objeto de estudio y metodológicamente considera injustificable la dicotomía radical entre ciencias de la naturaleza y ciencias del espíritu, ya que su metodología al ser neokantiana, muestra que para las diversas ciencias como objetos de conocimiento son fenoménicos, en el sentido kantiano.

Manuel Gil Antón sintetiza la metodología weberiana en los siguientes puntos, los cuales resumo a continuación:

a) Rechaza la dicotomía radical entre ciencias de la naturaleza y del espíritu, en especial lo incalculable de la acción social y la supuesta mensurabilidad absoluta de los hechos naturales. 
b) Las ciencias histórico-sociales, son ciencias y no deben reducir sus protocolos metodológicos a los establecidos por las ciencias de la naturaleza.

c) Las ciencias se distinguen entre sí por el interés cognitivo especifico ya sea por la explicación causal o por la comprensión interpretativa del sujeto cognoscente y no por el objeto de estudio. En el interés cognitivo de las ciencias de la acción social, la elaboración conceptual requiere una compleja relación entre el esquema causa-efecto y el esquema mediosfines, para llegar a la explicación comprensiva de los fenómenos.

d) En las ciencias histórico-sociales la elaboración de conceptos es relevante e implica una actividad del sujeto cognoscente, que selecciona elementos de la realidad relacionando los más importantes elementos de los fenómenos de estudio.

e) Los conceptos (tipos ideales) no son la realidad ni su copia, sino instrumentos de organización de la realidad elaborados por el sujeto cognoscente para poder explicar el fenómeno, esto permite al investigador contrastar la relevancia causal de diversos factores: «¿habría ocurrido lo mismo sin la presencia de determinado elemento? Si se afirma, su relevancia causal tiende a cero; si se niega, se puede establecer su relevancia causal en la generación del fenómeno a explicar comprensivamente» (Gil Antón, 2001; 621623).

En base a estas características metodológicas y en general las consideraciones weberianas para la explicación y comprensión sobre los suicidios, la 
investigación según Weber se centraría en la historia y psicología propia de cada individuo, intentando encontrar el sentido de su acción, buscando el significado de la acción de los individuos suicidas; negando la clasificación de Durkheim sobre los suicidios, pues estos se basan en el peso de la acción en común y no tanto en lo individual, además la caracterización del suicidio egoísta sería entendida como un concepto, un tipo ideal (un instrumento de investigación) para el estudio inquisitivo de los fenómenos observados y no una descripción de la realidad.

Así la clasificación de Durkheim de explicación del suicido: El suicido Egoísta, Altruista y Anómico, para Weber no serían más que elaboraciones de tipos ideales, necesarios para establecer las relaciones y correlaciones que llevarían a indagar la posible causa de su acción, faltando un análisis subjetivo de los motivos racionales o no de su acción. Así pues se observarían las estadísticas y se compararían los hechos con los tipos de suicidios elaborados teóricamente. De ahí que la posición de Weber se aproxima al instrumentalismo, pues todo conocimiento empírico sea cultural o natural está vinculado al instrumento de elaboración conceptual.

El positivismo ha permeado la idea de que es posible aplicar un método individualista, de hecho, ha tenido mucha aplicación en los modelos económicos ortodoxos, estos afirman que los fenómenos sociales pueden ser explicados por su reducción a los individuos. Por ejemplo en los modelos de la micro y la 
macroeconomía es común ver que la sociedad se reduce para su estudio a un consumidor o a una firma porque varios individuos, en este caso consumidores o empresas son supuestamente iguales entre un gran número de estas (Parada Corrales, 2004; 418). Bhaskar en cambio niega la reducción de las sociedades a individuos o a grupos (Bhaskar, 2005; 29-40).

Desde ahora es posible vislumbrar la problemática que existe en la constitución del individuo y de la sociedad. Desde lo cotidiano donde se ven los procesos de reproducción o transformación en la socialización, intrínsecamente relacionados. Durkheim comenta que la educación es un claro ejemplo de qué son los hechos sociales:

Además, podemos confirmar mediante una experiencia característica, esta definición del hecho social: basta observar la forma en que se educa a los niños. Cuando se observan los hechos tal como son y como han sido siempre, salta a la vista que toda educación consiste en un esfuerzo continuo por imponer al niño formas de ver, de sentir y de actuar a los cuales no llegaría espontáneamente (Durkheim, 2001; 43).

\section{El modelo transformacional de la actividad} Social (T.M.S.A)

Bhaskar desarrolla un modelo para el estudio de la actividad social, hace un análisis de los modelos 
elaborados por cuatro tendencias en el pensamiento social. Relaciona a Durkheim como colectivista en cuanto al objeto de estudio y en su método como empíricista; a Weber cómo individualista en el objeto de estudio y en su método como neokantiano; mientras que a Marx lo caracteriza como realista en su método y en cuanto a su objeto relacional.

Bhaskar caracteriza el modelo de Weber (modelo I) con el estereotipo de voluntarismo (el individuo constituye en gran medida la sociedad), y el modelo de Durkheim (modelo II) con la reificación (la sociedad constituye a los individuos). Agrega un tercer modelo dialéctico (modelo III), basado en P. Berger:

Según el modelo de Berger, que llamaré Modelo III, la sociedad forma a los individuos, quienes crean la sociedad; en otras palabras, la sociedad produce a los individuos, quienes producen la sociedad en una dialéctica continua ${ }^{3}$.

De acuerdo con este modelo es posible distinguir y hacer una categoría de los hechos sociales y de los naturales. Los sociales dependen esencialmente de la actividad humana, a la vez parece tener en cuenta tanto lo subjetivo e intencional, así como también lo

${ }^{3}$ According to the Berger model, which I shall call Model III, society forms the individuals who create society; society, in other words, produces the individuals, who produce society, in a continuous dialectic (Bhaskar 2005; 35). 
coercitivo de los hechos sociales mediando entre el modelo I y modelo II.

Bhaskar señala que este modelo (III) también es engañoso. «La razón es que la gente y la sociedad no pueden ser combinadas en dos momentos del mismo proceso. Son apenas cosas distintas» Da las siguientes razones de los fallos en los modelos mencionados: «Notice that on Model I there are actions, but no conditions; on Model II conditions, but no actions; on Model III no distinction between the two.» Es crucial observar que en el Modelo I hay acciones, pero no condiciones; en el Modelo II condiciones pero sin acciones; en el Modelo III no hay distinción entre los dos] $(2005 ; 40)$.

Así propone un cuarto modelo: Modelo Transformacional de la Actividad Social (Transformational Model of Social Activity, TMSA) El modelo transformacional de la actividad social, con el objetivo de dar una alternativa a las dicotomías que atraviesan el estudio social, centradas en la estructura/agencia y las divisiones presentes en su parte metodológica como es individualismo/holismo.

En este la sociedad y la acción del ser humano como individuo son a la vez la causa material de la sociedad y también la producción de la actividad humana. Como se puede observar en el modelo, las actividades y prácticas sedimentadas constituyen la socialización, es decir, que el individuo se encuentra insertado en un conjunto de actividades institucionalizadas que le preexisten, sin embargo, esas mismas prácticas existen más allá (además) de las 
estructuras y mecanismos por la acción de los individuos, ya sea reproduciendo y/o transformando dichas prácticas.

Model IV: The Transformational Model of the Society/Person Connection

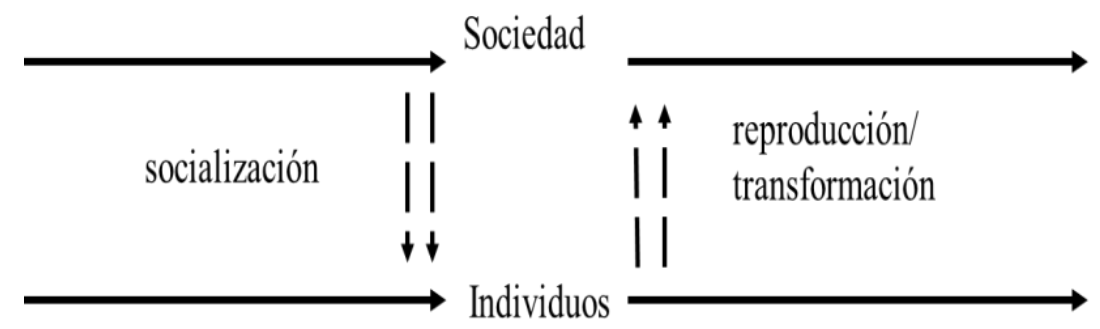

En el caso de la sociedad las estructuras están cargadas de significado, y suscribe la tesis de la doble hermenéutica, esto es que las ciencias sociales (el sujeto cognoscente) son la parte de su mismo objeto de estudio, sin embargo el aceptar esto para el realismo crítico no implica negar el carácter de intransitividad, lo que quiere decir que las estructuras sociales: «existen con independencia de los individuos, aunque están mediadas por la agencia» (Murguía, 2006; 75).

De manera que la agencia es la condición necesaria para la posibilidad de una estructura, pero la estructura misma es de una ontología distinta a la de la agencia, pues posee una realidad sui generis, por lo que no es reductible a los individuos: «una vez que la sociedad existe, existe independientemente de cómo se haya producido, constituye una dimensión intransitiva de la realidad susceptible de ser explicada científicamente» (Murguía, 2006; p. 75). El que sea parte de la dimensión 
intransitiva de la realidad no implica que no sea de carácter histórico, por ende es susceptible de transformación.

Un ejemplo de esto es el lenguaje como estructura social, este existe por la prácticas de los individuos, aunque también, la estructura del lenguaje le preexiste a los individuos los individuos con sus prácticas van transformando o reproduciendo las estructura del lenguaje y lo mismo sucede con diversos fenómenos sociales, lo interesante aquí es la naturaleza intransitiva de las estructuras sociales, lo que Bhaskar menciona como los dos lados del conocimiento es para afirmar lo siguiente: las estructuras sociales son existencialmente independientes de nuestro conocimiento, pero las estructuras son causalmente interdependientes con el conocimiento.

El modelo de Durkheim presenta limitantes a la agencia y por el contrario, el modelo de Weber presenta mayor libertad al individuo. Por eso pareciera que el modelo del construccionismo social (P. Berger), hace justicia a los dos modelos anteriores, pero no hace la distinción ontológica entre estructura y agencia, de tal forma el modelo de Berger elabora una dialéctica entre agencia y estructura, donde los individuos son constituidos por la sociedad y a su vez la sociedad por los individuos. Bhaskar comenta que el modelo de Berger toma valor tanto del aspecto subjetivo e intencional del modelo weberiano, como lo coercitivo y externo del modelo de Durkheim, pero el problema es la distinción ontológica de la estructura frente a la agencia, pues son objetos similares pero distintos. 
El modelo de Bhaskar es relacional, es decir, los agentes no crean la estructura social, como lo propone el constructivismo, más bien, opta por la reproducción o transformación de la estructura, pues ésta les antecede. Esta concepción de la estructura cambia la discusión típica en la conexión de sociedad y persona puesto que la estructura preexiste al individuo. El modelo de Bhaskar es relacional, con esto quiere decir que las personas no son producto de la sociedad, las relaciones de los distintos tipos de mecanismos y estructuras constituyen la sociedad, que aunque dependen de la actividad humana no se pueden reducir a las prácticas de las personas puesto que también hay mecanismos emergentes tanto materiales como no materiales.

El TMSA considera los procesos de reproducción y cambio de las estructuras sociales, así la estructura social no es únicamente alienante, la sociedad no se puede concebir como un producto de decisiones humanas incondicionadas por las restricciones, limitantes y oportunidades heredadas del pasado y el entorno. Más bien, el TMSA opta por una sociedad donde las personas conscientemente transforman sus condiciones sociales de existencia, es decir a su estructura, inician o constituyen rupturas, mutaciones o, en general, transformaciones.

Parada comenta sobre la posición de Bhaskar sobre el carácter de emergencia, con la relación de la mente con el cerebro, procede de manera similar con la sociedad en relación con la praxis de los individuos. 
Pero en cuanto al comportamiento, Bhaskar también critica el reduccionismo material en psicología.

Es verdad que sin cerebro no hay mente. Pero la mente humana emerge del cerebro. No puede ser reducida a éste [...]. La mente es una entidad sui géneris, una clase en sí misma. Cuando la mente emerge del cerebro adquiere una existencia independiente; puede incluso alterar la dimensión material del cerebro en sí mismo. Cuando las personas actúan, están interviniendo causalmente en el mundo material. Sin embargo, su accionar está sujeto a la timidez reflexiva. Estos actos podían ser o no intencionales (Bhaskar ,2004; 421- 422).

De esta manera, el naturalismo crítico implica un avance, ya que el naturalismo tradicional no reconoce los poderes de los agentes, es decir, sus poderes causales; y por el otro lado, las sociologías comprensivas no reconocen los poderes causales de las estructuras sociales, y tampoco como en la teorías de la mutua constitución, así también no implica una dualidad estructural sociedad-individuo, sino que, corresponden a realidades distintas y su justificación depende de disciplinas distintas: los poderes de los agentes a la psicología y los de la sociedad a la sociología. Bhaskar caracteriza a las estructuras sociales distintas a las de la ciencias naturales, por eso su propuesta ha sido llamada naturalismo cualificado, puesto que al pertenecer las estructuras sociales a la dimensión intransitiva es posible el estudio científico 
de la estructuras y mecanismos sociales tomando en cuenta las siguientes características propias de las de los social.

1. Las estructuras sociales, a diferencia de las estructuras naturales, no existen independientemente de las actividades que gobiernan.

2. Las estructuras sociales, a diferencia de las estructuras naturales, no existen independientemente de las concepciones de los agentes sobre lo que están haciendo en su actividad.

3. Las estructuras sociales, a diferencia de las estructuras naturales, pueden ser relativamente duraderas (de modo que las tendencias que fundamentan pueden no ser universales en el sentido de ser invariantes en espacio-tiempo $)^{4}$.

\section{La mutua constitución y la dualidad de la estructura}

A partir de la segunda mitad del siglo XX la Teoría Social tuvo bastante desarrollo con intensiones

4 1. Social structures, unlike natural structures, do not exist independently of the activities they govern.

2. Social structures, unlike natural structures, do not exist independently of the agents' conceptions of what they are doing in their activity.

3. Social structures, unlike natural structures, may be only relatively enduring (so that the tendencies they ground may not be universal in the sense of space-time invariant) (Bhaskar, 2005; 42). 
sintéticas entre la estructura y la agencia, como pretendía la sociología Parsoniana, ejemplo de esto son las teorías sociológicas que surgen desde la década de los setentas en adelante, como son: A. Touraine, J Alexander, P. Bourdieu y A. Giddens, que entienden la sociedad de manera sintética, que supere la dicotomía entre individualismo y holismo, superando la escisión entre el colectivo y las acciones individuales, de tal manera que la sociedad no es independiente de los individuos que la componen ni los individuos de las relaciones colectivas. A esto se le ha llamado de manera general la mutua constitución. Las teorías sociales que tratan de superar las dicotomías que traen consigo «las tradiciones naturalistas y compresiva: individuosociedad / subjetivismo-objetivismo / acciónestructura (o sistema)» (Murguía, 2006; 61).

Lo anterior ha producido debates que tienen implicaciones ontológicas sobre el objeto de estudio de las ciencias sociales, ¿Cuál es la realidad del objeto que se pretende estudiar?, ¿A qué se refiere cuando se habla de naturaleza humana?, ¿Qué tipo de entidad es la sociedad?

Para A. Giddens el objeto de estudio primario para las ciencias sociales en su teoría de la estructuración no implica ni la vivencia del actor individual, ni la existencia de alguna forma de totalidad societaria, sino que se debe a prácticas sociales ordenadas en un espacio y un tiempo determinado. La continuidad de prácticas genera reflexividad pero esta última solo es posible a partir de la continuidad de dichas prácticas (Giddens, 2006; 40). 
A partir de esto Giddens formula un modelo estratificado del agente, el agente entendido como un actor reflexivo que supone diversas estratificaciones de su obrar. En su modelo el papel del agente es notorio y central puesto que no hay cambio sin reflexividad, esta es entendida como una racionalización en proceso, más que como un estado, pues parte de la intrínseca competencia de agentes que parten de una temporalidad, es así como estructuración tiene un cierto sentido de historia. Entendiendo aquí la estructura como un conjunto de reglas y recursos en el conjunto de relaciones de transformación. Giddens más allá de entender estructura a modo de la tradición estructuralista, los sistemas sociales tiene propiedades estructurales o sistémicas, por lo que en su teoría de la estructuración los sistemas son relaciones reproducidas por los actores o colectividades de manera regular (Giddens, 2006; 61). Con lo anterior la estructura es la vez que constrictiva habilitadora tal y como el modelo TMSA del realismo crítico.

Giddens se enfoca en las características del agente, en especial sobre su reflexión. Como veremos a continuación, la teoría de Giddens carece de análisis sobre la acción colectiva. Giddens hace una crítica tanto a las concepciones del estructuralismo como al funcionalismo de insistir en el vigor de la estructura, si no es con la existencia de un todo social, al menos con la preeminencia del colectivo sobre sus partes.

Es por esto que Giddens propone tres conceptos sobre los cuales fundamenta su teoría social en lo que llama dualidad de estructura, el sistema (como realidad 
institucionalizada la cual se reproduce o transforma), estructura (un orden virtual de reglas y recursos que moldean la acción), la estructuración (entendida como las condiciones que mantiene dicha reproducción o transformación no solamente de las estructuras sino también del sistema mismo) (King, 2010).

Giddens reconoce que las actividades humanas están ordenadas en un espacio y un tiempo, bajo el punto de vista hermenéutico es esencial que la actividades humanas están cargadas de significación o intención, pero la reflexividad a la que se refiere Giddens «no se debe entender como mera "auto-conciencia" sino como el carácter registrado del fluir de una vida social» (Giddens, 2006), es así como el modelo de estratificación representa el registro reflexivo de la acción cotidiana del individuo y de los otros.

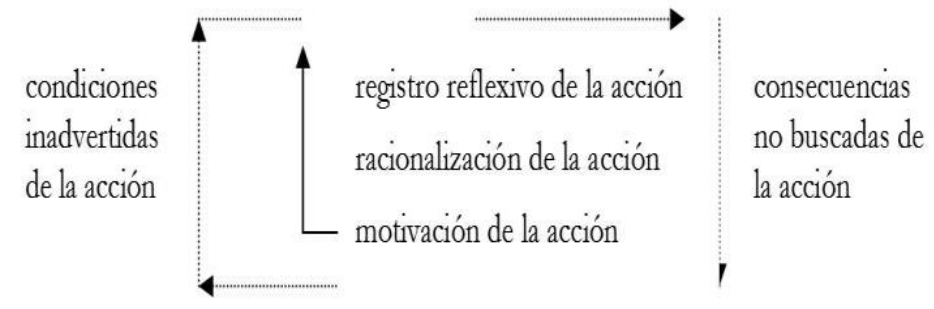

La motivación de la acción denota los deseos que la mueven y de nuestra conducta cotidiana, en gran medida no se reconoce una motivación directa, esto es, que la motivación se entiende como lo inconsciente, de manera diferente al psicoanálisis, más bien entendida como la cognición inconsciente. Después viene la racionalización de la acción a la que se refiere una 
conciencia práctica (una comprensión de la rutina de la acción), y por último el registro reflexivo de la acción, la cual archiva actividades y crea expectativas de las acciones de los otros. Además ofrece un análisis de como dichas relaciones entre los conjuntos de acciones no buscadas generan consecuencias inadvertidas de la acción. Donde la acción produce las reglas y estas los recursos para la reproducción de la acción.

Giddens ejemplifica con una distribución urbana donde individuos de diversos grupos étnicos no quieren vivir en un espacio donde se encuentre siendo minoría, por lo que sus acciones intencionales o no constituyen reglas como consecuencias no buscadas relacionadas por condiciones inadvertidas. De tal modo que los resultados de vivir en un cierto mercado de vivienda se lleva acabo de manera intencional. «Pero el resultado final no fue buscado ni deseado por nadie. Es, por así decir, obra de todos y de nadie» (Giddens, 2006; 43-47).

A partir de la teoría de Giddens cabe señalar lo siguiente, a propósito de la estructuración: a) la estructura es virtual, es decir no existe, sino que es actuada por el agente, b) el agente constituye con su actuar modos de acción que se fundamentan en su naturaleza estableciendo reglas y c) posteriormente se institucionalizan por medio de los recursos. De esta forma la estructura social es habilitadora o constrictiva de la acción. 


\section{Estructura, virtual o emergente}

El enfoque metodológico del realismo crítico de Bhaskar ha tenido relevancia, ya sea para la filosofía de la ciencia en general $o$ en distintas disciplinas científicas, como son el caso de ciencias sociales, como sociología, economía y derecho, por ejemplo en sociología se encuentra en el enfoque morfogenético de Margaret Archer; en economía se encuentra Tony Lawson. El trabajo sociológico de M. Archer se fundamenta en la ontología desarrollada por Bhaskar y en gran medida por el modelo transformacional de la actividad social.

Con respecto a la teoría de Giddens se han señalado que la estructura entendida como virtual representa críticas en cuanto a estado y función como estructura virtual, puesto que tiene implicaciones importantes, ya que el modelo de Giddens fusiona el agente humano con el sistema. En otras palabras, la teoría de la estructuración sobreestima la libre capacidad del individuo, ya que lo constitutivo de la estructura son las reglas que se pueden reproducir o no, además desdeña la fortaleza que implica la preeminencia del colectivo ante el individuo: la subestimación sistemática de las limitaciones infla artificialmente los grados de libertad de acción ${ }^{5}$.

5 «the systematic underplaying of constraints artificially inflates the degrees of freedom for action» (Archer, 2010; 234). 
La socióloga Margaret Archer, quien comenta que el enfoque morfogenético (1995), complementa el modelo TMSA, propuesto por Bhaskar, como ya se mencionó el modelo TMSA, presenta un carácter dual del estructura y de la agencia, entendidas como praxis humana, como distintas entidades y distintos niveles ontológicos pero que son interdependientes causales. Archer se basa en los siguientes principios del TMSA:

1) La sociedad no puede reducirse a las personas,

2) la sociedad preexiste a las personas $y$, por ende, es un objeto de investigación independiente,

3) la sociedad tiene poderes causales que se identifican con el dominio de lo real y

4) los poderes causales de la sociedad están mediados por la actividad humana.

(Archer, 1995).

La aportación principal de Archer en cuanto al modelo de Bhaskar es insertar como categoría al Tiempo, para poder presentar la distinción en la estructura (preexistente), con las prácticas que corresponden a la agencia, que puede reproducir o transformar dicha estructura. Con esto pretende identificar las continuidades y discontinuidades temporales entre las acciones de las estructuras y de las agencias, para esto menciona tres etapas para su análisis: i) condicionamiento, ii) interacción y iii) elaboración. 
En la etapa de interacción tiene el primer momento la relación entre propiedades emergentes materiales con las propiedades emergentes culturales, de este primer momento surge un segundo orden de propiedades emergentes, así Archer menciona que se dan las siguientes situaciones lógicas:

1. Lógica de la protección, donde lo material y lo cultural coinciden, favoreciendo así una expectativa de reproducción de las estructuras sociales.

2. Lógica del compromiso, la cual recrea una incompatibilidad cultural-material donde, básicamente, los deseos de cambio de la sociedad (intrínsecos en motivaciones culturales preexistentes) no son lo suficientemente fuertes para materializarse.

3. Lógica del oportunismo, donde surgen contextos materiales y culturales contingentes (ej. una guerra o el surgimiento de un nuevo paradigma científico) que motivan a algunos actores sociales a asociarse para desafiar las estructuras dominantes.

4. Lógica de la eliminación, en la cual las condiciones materiales y culturales, también contingentes, favorecen los intereses de algunos grupos para eliminar por completo a sus adversarios políticos o económicos y modificar el régimen a su conveniencia (Parra-Heredia, 2016; 226).

En la etapa de elaboración se refiere a la constitución de las estructuras por medio de los actores que pueden 
contribuir a la reproducción (morfostasis) o bien la transformación (morfogénesis) del sistema ya existente. A partir de esto se pueden tipificar cuatro escenarios posibles:

i) morfostasis material y cultural, ii) morfogénesis material y cultural, iii) morfostasis material y morfogénesis cultural, y iv) morfogénesis material y morfostasis cultural (Parra-Heredia, 2016; 227).

El punto focal en la discusión entre A. Giddens y M. Archer junto con la ontología del realismo crítico es el carácter emergente de lo social. Las estructuras sociales y las personas se entienden en el naturalismo crítico como entidades distintas, las cuales operan en niveles ontológicos distintos.

Cabe destacar del modelo Bhaskariano, a diferencia del de la estructuración de Giddens la relación entre sociedad y la persona, presupone una preexistencia de las estructuras, esto es que las personas surgen en medio de un contexto social, como son: la familia, las normas sociales de la cultura, tradiciones, instituciones y lenguajes (condiciones).

El modelo de conexión sociedad / persona que propongo podría resumirse de la siguiente manera: las personas no crean la sociedad. Porque siempre los preexiste y es condición necesaria para su actividad. Más bien, la sociedad debe considerarse como un conjunto de estructuras, prácticas y convenciones que los 
individuos reproducen o transforman, pero que no existirían a menos que lo hicieran ${ }^{6}$.

Archer señala la conflación de la teoría de la estructuración de Giddens señalando dos asuntos centrales: a) la estructura preexiste a los individuos ya que se encuentra en algún espacio-tiempo determinado, y en segundo lugar b) las acciones transformadoras se ubican en un tiempo distinto al de las condiciones constrictivas de dichas acciones:

[...] la afirmación de Giddens de que «los sistemas sociales sólo existen mediante su estructuración continua en el tiempo» La preexistencia y autonomía refieren a discontinuidades en el proceso de estructuración/reestructuración que solo pueden capturarse al hacer distinciones analíticas entre el antes (Fase 1), el durante (Fase 2) y el después (Fase 3), lo que no niega la necesaria continuidad de la actividad humana para la durabilidad de todo aquello que es social (Archer, 1995; 200).

${ }^{6}$ The model of the society/person connection I am proposing could be summarized as follows: people do not create society. For it always preexists them and is a necessary condition for their activity. Rather, society must be regarded as an ensemble of structures, practices, and conventions which individuals reproduce or transform, but which would not exist unless they did so (Bhaskar, 2005; 39). 
El modelo transformacional de la actividad social, así como el enfoque morfogenético permiten un análisis completo de las estructuras, incluso de su ontología como preexistente y emergente por lo que explica de manera más profunda las relaciones entre individuos de las condiciones constrictivas y las acciones transformadoras, esto es entender ambas, condiciones $\mathrm{y}$ acciones como acciones condicionadas.

\section{Bibliografía}

Archer, M. S. (1995). Realist Social Theory. Cambridge University Press.

https://doi.org/10.1017/CBO9780511557675

Archer, M. S. (2010). Morphogenesis versus structuration: on combining structure and action1. The British Journal of Sociology (61), 225252.

Bhaskar, R. (2005). The Possibility of Naturalism, A Philosophical Critique of the Contemporary Human Sciences. Philosophical Books (22).

Bhaskar, R. (2008). A Realist Theory of Science. New York:

Routledge. https://doi.org/10.2307/2184170

Durkheim, E. (2001). Las Reglas Del Método Sociológico.

(E. Champourcín, Ed.). México: FCE.

Durkheim, E. (2014). El Suicidio. México: Grupo Tomo. Giddens, A. (2006). La constitución de la sociedad: bases para la teoria de la estructuración. (J. L. Etcheverry, Ed.) (1a ed.). Amorrortu.

Gomez Sandoval, F. (1986). Historia de la Sociología (2a. 
ed.). México: Costa-Amic Editores.

Gil Antón, M. (2001). En torno a la propuesta weberiana: el papel de lo imprevisto. Estudios Sociológicos De El Colegio De México.

Gómez Rodríguez, A. (2003). Filosofía y metodología de las ciencias sociales. Madrid: Alianza Editorial.

King, A. (2010). The odd couple: Margaret archer, Anthony Giddens and British social theory. British Journal of Sociology (61), 253-260.

Murguía, A. (2006). Perspectivas y Horizontes de la filosofia de la ciencia a la vuelta del tercer milenio. Volumen III. México: Universidad Nacional Autónoma de México.

Parada Corrales, J. (2004). Realismo crítico en investigación en ciencias sociales: una introducción. Investigación \& Desarrollo (12), 396429.

Parra-Heredia, J. (2016). Realismo crítico: una alternativa en el análisis social. Sociedad y economía (31), 215-238.

Roy Bhaskar, Margaret Archer, Andrew Collier, Tony Lawson, A. N. (2001). Critical Realism: Essential Readings. Historical Materialism, 8(1).

Weber, M. (1964). Economía y Sociedad. Esbozo de sociología comprensiva. Madrid: FCE. 